[CONTRIBUTION FROM THE, JOHN HARRISON LABORATORY OF CHEMISTRY. No. [3.]

\title{
DETERMINATION OF THE ATOMIC MASSES OF SILVER, MERCURY AND CADMIUM BY THE ELECTRO. LYTIC METHOD. ${ }^{1}$
}

\author{
BY WILLETT IEPLEY HARDIX. \\ Received Septernber 26,1896 .
}

INTRODUCTION.

\begin{abstract}
A glance at the literature on the determinations of the $A$ atomic masses of silver, cadmium and mercury will show that, with the exception of cadmium, the electrolytic method has not been tried. Aside from the fact that certain errors involved in the washing and drying of the precipitates are eliminated by this method, its simplicity at once gives it preference over the usual methods of gravimetric determinations. Inasmuch as these three metals are completely precipitated from certain of their solutions by the electric current, and as it is desirable to determine the atomic mass of any element by different methods, it was thought advisable to apply this method in a redetermination of the atomic masses of these elements.
\end{abstract}

GENERAI CONSIDERATIONS.

Before taking up the different metals separately, the following general considerations may be mentioned:

I. A careful preliminary study was made in the selection of compounds. Some compounds, which from a theoretical standpoint seemed to offer certain advantages, were found by experiment not to meet the requirements of exact determinations. Salts which can be sublimed were used whenever possible; and in all cases only those salts were used which form well defined crystals.

2. All reagents used were either prepared or purified by myself and carefully tested for impurities.

3. The metals were deposited in platinum dishes of about 200 cc. capacity and about sixty-five grams in weight. When the precipitation was complete, before interrupting the current, the

1 From the author's thesis presented to the Faculty of the University of Pennsylvania for the degree of $\mathrm{Ph} . \mathrm{D}$., I 896 . 
solution was siphoned from the platinum dish, pure water being added at the same time; this was continued until the solvent used was completely removed from the dish. The current was then interrupted and the deposit washed several times with boiling water, with the hope of removing any accluded hydrogen. After drying, the dishes were placed in a vacuum desiccator over anhydrous calcium chloride and allowed to remain in the balance room until their temperature was the same as that of the room. Atmospheric dust was excluded from the platinum dishes during the process of deposition by means of two glass plates which formed a complete cover; the moisture which collected on this cover was washed back into the dish from time to time. The dishes were handled with nickel tongs tipped with rubber.

4. The balance used was made expressly for this work by Henry Troemner, of Philadelphia. The beam and pans were made of aluminum, the beam being about twenty centimeters long. The framework was plated with gold to prevent corrosion. The sensibility for different loads and the ratio of the length of the two arms were carefully determined. The balance is sensitive to the fortieth of a milligram, and the sensibility is almost independent of the load up to seventy-five grams. The difference in the length of the two arms is so slight that no correction need be applied. The balance was kept in a large quiet room of nearly constant temperature.

The larger weights used were made of brass and the fractions of a gram made of platinum. The weights were all previously compared against each other and standardized with reference to the largest weight. The small corrections found in comparing them were tabulated and applied to all results. The weighings were made by the method of oscillations. The temperature and barometic pressure were noted at the time of each weighing, and all weighings were reduced to a vacuum standard. As the density of the atmosphere at the time of weighing the empty platinum dish was different from that at the time of weighing the dish and deposit together, the following formula was applied to obtain the weight of the deposit in vacuo: 


$$
\left[\begin{array}{c}
{\left[\text { Weight of }(\text { dish }+ \text { deposit })-\frac{\text { weight of } \operatorname{dish}\left(1+\frac{\lambda}{\Delta}-\frac{\lambda}{f}\right)}{1+\frac{\lambda^{\prime}}{\Delta}-\frac{\lambda^{\prime}}{f}}\right]} \\
\times\left[\mathrm{I}+\frac{\lambda^{\prime}}{\Delta^{\prime}}-\frac{\lambda^{\prime}}{f}\right]=\text { weight of deposit in vacuo. }
\end{array}\right.
$$

Where $\lambda=$ density of air at the time of weighing the empty dish.

$\lambda^{\prime}=$ density of air at the time of weighing the dish + deposit.

$\Delta=$ density of platinum dish.

$\Delta^{\prime}=$ density of metallic deposit.

$f=$ density of weights.

As the weights were all standardized with reference to the hundred-gram brass weight, it is evident that they must all be calculated as having the same density, equal to that of brass.

5. The atomic masses of the different elements involved in the calculation of results were taken from Clarke's latest report. ${ }^{1}$

\section{PART I.}

DETERMINATION OF THE ATOMIC MASS OF SILVER.

The mean of all the earlier determinations, as calculated by Clarke. gives 107.923 for the atomic mass of silver; a result almost identical with the mean (107.93; $\mathrm{O}=16$ ) of the determinations of Stas.

\section{PREPARATION OF PURE METALIIC SILVER.}

The silver used in this work was purified by the Stas method. Two hundred grams of silver, about ninety-nine per cent. pure, were dissolved in dilute hot nitric acid. The solution was evaporated to dryness, the nitrate heated to fusion and maintained in a fused condition until the oxides of nitrogen were no longer evolved. The residue, after cooling, was dissolved in as little cold water as possible, and after standing forty-eight hours the solution was filtered through a double filter to remove any suspended matter. The clear solution was then diluted with thirty times its volume of distilled water, and to it was added an

1J. Am. Chem. Soc., 18, 197. 
excess of pure hydrochloric acid. The silver chloride which separated was allowed to subside and was then thoroughly washed by decantation, at first with water containing a little hydrochloric acid, and finally with pure water. The precipitate was then collected on a cheese cloth filter, pressed strongly and allowed to dry. When perfectly dry, the silver chloride was powdered finely and digested for three days with aqua regia; it was then thoroughly washed by decantation with distilled water. After obtaining the pure chloride of silver, it was necessary to purify the caustic potash and milk sugar used in reducing the chloride to the metallic state. The caustic potash was heated to the boiling point and to it was added a concentrated solution of potassium sulphide to precipitate any leavy metals which might be present. The solution was filtered and the filtrate digested for some time with freshly precipitated silver oxide and again filtered to remove the excess of potassium sulphide. The milk sugar was purified in a similar manner. The silver chloride was then placed in large porcelain dishes and covered with a solution of caustic potash and milk sugar. The dishes were placed on a water-bath and heated to a temperature of $70^{\circ}-80^{\circ}$ until the reduction to finely divided metallic silver was complete. The alkaline solution was then poured off, and the gray metallic silver was washed with distilled water until the alkaline reaction disappeared. The metal was then digested with pure dilute sulphuric acid, and finally washed with dilute ammonia water. The silver thus obtained was mixed, when dry, with five per cent. of its weight of fused borax containing ten per cent. of pure sodium nitrate. The mixture was fused in a clay crucible and the silver poured into a mold. The metal obtained in this way was almost snow white in appearance, and dissolved completely in nitric acid to a colorless solution.

PREPARATION OF PURE NITRIC ACID.

To obtain pure nitric acid, one-half liter of the commercial C. P. acid was mixed with an equal volume of concentrated C. P. sulphuric acid and distilled from a retort provided with a knee tube and condenser. The first portion of the distillate was rejected. The process was stopped when half of the 
nitric acid present had been distilled over. The distillate was mixed with an equal volume of pure sulphuric acid and redistilled. The second distillate was collected in a flask, the mouth of which was closed with glass wool. When the process was complete, the flask was closed with a doubly perforated cork and placed in a water-bath at a temperature of $40^{\circ}$. A current of pure dry air was then conducted through the acid to remove any oxides of nitrogen. The acid was kept in a dark place.

\section{EXPERIMENTS ON SILVER OXIDE.}

If pure, dry silver oxide could be prepared, the atomic mass of silver could be compared directly with that of oxygen. A large number of experiments were made on this compound with the hope of determining the ratio of the atomic masses of these two elements.

\section{PREPARATION OF SILVER OXIDE.}

A portion of the pure metallic silver was dissolved in pure dilute nitric acid and the solution evaporated to crystallization. The crystals of silver nitrate were dissolved in pure water and to the solution was added a solution of pure sodium hydroxide, prepared by throwing pieces of metallic sodium on distilled water in a platinum dish. The twenty-five grams of silver oxide prepared in this way were washed by decantation with twenty liters of water. The material was then dried at the ordinary temperature, after which it was finely powdered and dried for twenty-four hours in an air-bath at $100^{\circ}$. The oxide was kept in a weighing tube in a dark place.

Several analyses were made by dissolving a weighed portion of the material in pure potassium cyanide, electrolyzing the solution and weighing the resulting metallic silver. The observations invariably gave less than ninety-five for the atomic mass of silver. The oxide was redried at a temperature of $125^{\circ}$ and analyzed as before, but the quantity of silver obtained was far below that calculated for the compound $\mathrm{Ag}_{2} \mathrm{O}$. Observations were also made on material dried at $140^{\circ}$ and $150^{\circ}$. The results showed that it was impossible to prepare the silver oxide in a pure, dry condition. 
After making these observations, my attention was called to an article by $M$. Carey Lea, ' in which were given the results of a series of analyses of silver oxide dried at different temperatures varying from $I 00^{\circ}$ to $I 70^{\circ}$. These observations prove conclusively that oxygen is given off at a much lower temperature than that required to remove the last traces of moisture. From these observations and the results obtained by myself, it was evident that any further attempt to determine the atomic mass of silver from the oxide would be useless.

Although no careful study was made as to the nature of this compound, it might be added that, from my own observations, it seems very probable that the oxide contains some hydrogen in the form of hydroxyl.

\section{FIRST' SERIES.}

\section{EXPERIMENTS ON SILVER NITRATE.}

The nitrate of silver seems to fulfil the conditions necessary for accurate analyses, inasmuch as it is stable and crystallizes in well defined crystals which can be fused without decomposition.

\section{PREPARATION OF SII.VER NITRATE.}

The material used in these experiments was prepared by dissolving pure silver in pure aqueous nitric acid in a porcelain dish. An excess of silver was used, and after complete saturation the solution was poured off from the metal into a second dish and evaporated to crystallization. The perfectly transparent, rhombic plates of silver nitrate which separated were dissolved in pure water and recrystallized. The crystals were then carefully dried, placed in a platinum crucible which rested in a larger platinum dish and gradually heated to fusion. After cooling, the perfectly white opaque mass was broken up and placed in a ground-glass stoppered weighing tube and kept in a desiccator in a dark place.

MODE OF PROCEDURE.

The platinum dish in which the deposit was made was careI Am.J. Sci., 44, 240. 
fully cleaned with nitric acid and dried to constant weight. It was then placed in a desiccator over anhydrous calcium chloride, and this, together with the desiccator containing the tube of silver nitrate, was placed in the balance room, where they were allowed to remain until their temperatures were the same as that of the room. After weighing the platinum dish, the tube of silver nitrate was weighed and part of the salt removed to the dish, after which the tube was reweighed. The difference in the two weighings, of course; represented the weight of silver nitrate used in the experiment. Enough water to dissolve the nitrate was added to the dish, and then a solution of potassium cya. nide, made by dissolving seventy-five grams of pure potassium cyanide in one liter of water, was added until the silver cyanide first formed was completely dissolved. The dish was then filled to within a quarter of an inch of the top with pure water and the solution electrolyzed with a gradually increasing strength of current. The following table will show the strength of current and the time through which it acted:

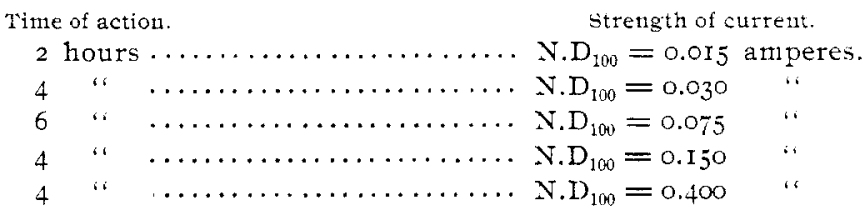

By gradually increasing the strength of current in this way the silver came down in a dense, white deposit. When the deposition was complete, before interrupting the current, the liquid was siphoned from the dish, pure water being added at the same time. This was continued until the cyanide was completely removed. The dish with the depasit was washed several times with boiling water and carefully dried. It was then placed in a desiccator and allowed to remain in the balance room until its temperature was the same as that of the room, when it was reweighed.

Weight of platinum dish $=71.27302$ grams.

Weight of silver nitrate $=0.31198$ grams.

Temperature, $22^{\circ}$.

Barometric pressure, $77^{\circ} \mathrm{mm}$.

Weight of platinum dish + silver deposit $=7$ 1.47ro4 grams. 
ATOMIC MASSES OF SILVER, MERCURY AND CADMIUM. 997

Temperature, $22^{\circ}$.

Barometric pressure, $760 \mathrm{~mm}$.

Density of silver nitrate $=4.328$.

" "brass weights $=8.5$.

" " platinum dish $=2 \mathrm{I} .4$.

" " metallic silver $=$ I0.5.

" " atmosphere at the time of weighing the empty dish and silver nitrate $=0.0012 \mathrm{I} 2$.

" " atmosphere at the time of weighing the platinum dish + silver deposit $=0.00$ I 96 :

Computing on this basis we have the following :

$0.31198\left(\mathrm{I}+\frac{0.0012 \mathrm{I} 2}{4.328}-\frac{0.001212}{8.5}\right)=0.31202=$ weight of $\mathrm{AgNO}_{3}$ in vacuo.

$7.27302\left[\frac{I_{1}+\frac{0.001212}{2 I .4}-\frac{0.001212}{8.5}}{L I+\frac{0.001196}{21.4}-\frac{0.001196}{8.5}}\right]=7 \mathrm{I.27291}=$ weight of platinum dish at $22^{\circ}$ and $760 \mathrm{~mm}$.

$71.47104-71.2729 \mathrm{r}=0.198 \mathrm{I} 3=$ weight of deposit at $22^{\circ}$ and $760 \mathrm{~mm}$.

0.198 I $3\left(\mathrm{r}+\frac{0.001196}{10.5}-\frac{0.001196}{8.5}\right)=0.19812=$ weight of deposit in vacuo.

Taking $\mathrm{O}=\mathrm{I} 6$ and $\mathrm{N}=\mathrm{I} 4.04$, the atomic mass of silver $=$ $\frac{0.19812 \times 62.04}{(31202-19812)}=107.914$.

Ten observations on silver nitrate computed in the foregoing manner are as follows :

$\begin{array}{cccc} & \begin{array}{c}\text { Weight of } \mathrm{AgNO}_{3} . \\ \text { Gram. }\end{array} & \begin{array}{c}\text { Weight of Ag. } \\ \text { Gram. }\end{array} & \begin{array}{c}\text { Atomic mass } \\ \text { of silver. }\end{array} \\ \text { I } & 0.3 \mathrm{I2O2} & 0.198 \mathrm{I} 2 & \\ 2 & 0.47832 & 0.30370 & 107.914 \\ 3 & 0.56742 & 0.36030 & 107.900 \\ 4 & 0.57728 & 0.36655 & 107.923 \\ 5 & 0.69409 & 0.44075 & 107.914 \\ 6 & 0.86367 & 0.54843 & 107.935 \\ 7 & 0.868 \mathrm{II} & 0.55 \mathrm{I} 30 & 107.932 \\ & & & 107.960\end{array}$




\begin{tabular}{|c|c|c|c|}
\hline & $\begin{array}{l}\text { Weight of } \mathrm{AgNO}_{3} . \\
\text { Gram. }\end{array}$ & $\begin{array}{c}\text { Weight of } A g . \\
\text { Gram. }\end{array}$ & $\begin{array}{l}\text { A tomic mass } \\
\text { of silver. }\end{array}$ \\
\hline 8 & 0.93716 & 0.59508 & 107.924 \\
\hline 9 & I.06170 & 0.67412 & 107.907 \\
\hline \multirow[t]{6}{*}{ To } & I. 19849 & 0.76104 & 107.932 \\
\hline & Mean & $=107.924$ & \\
\hline & Maximum & $=107.960$ & \\
\hline & Minimum & $=107.900$ & \\
\hline & Difference & $=0.060$ & \\
\hline & Probable er & $= \pm 0.005$ & \\
\hline
\end{tabular}

Computing the atomic mass of silver from the total quantity of material used and metal obtained, we have 107.926.

SECOND SERIES.

EXPERIMENTS ON SILVER ACETATE.

The fact that silver forms well crystallized salts with a number of organic acids nakes the comparison of the atomic mass of silver with the combined atonic masses of carbon, hydrogen, and oxygen, a matter of no great difficulty. From certain preliminary experiments, the acetate of silver seemed to fulfill the conditions necessary for accurate determinations.

PREPARATION OF SILVER ACETATE.

The purest commercial sodium acetate was dissolved in water, the solution filtered and recrystallized. After three crystallizations the material was dissolved in pure water, and to the rather concentrated solution was added a solution of silver nitrate, prepared in the manner already indicated. The white curdy precipitate which separated, after washing with cold water, was dissolved in hot water, the solution filtered and evaporated to crystallization. The silver acetate separated in brilliant swordshaped crystals. After pouring off the solution the crystals were quickly rinsed with cold water and placed between filters to remove the adhering moisture. The material was allowed to remain in contact with the filters only for a short time. It was then placed in a platinum dish, and when apparently dry the crystals were broken up into a finely divided condition and dried forty-eight hours in a vacuum desiccator. This work was carried on in a darkened room, and the silver acetate obtained was 
ATOMIC MASSES OF SILVER, MERCURY AND CADMIUM. 999

placed in a weighing tube, and kept in a desiccator in a dark place.

\section{MODE OF PROCEDURE.}

The method of operation was similar to that described under silver nitrate. After weighing the silver acetate, its aqueous or cyanide solution was electrolyzed and the weight of the resulting metallic silver determined. The results obtained from the aqueous solution were sometimes vitiated by the separation of silver peroxide at the anode. To prevent this, potassium cyanide was sometimes added. The results, however, from the two solutions were practically the same when no peroxide separated. From the aqueous solution the silver was deposited in a crystalline form. The strength of current and time of action were the same as for silver nitrate.

Ten observations on silver acetate reduced to a vacuum standard on the basis of

$$
\begin{aligned}
& 3.24 \mathrm{I}=\text { density of silver acetate, } \\
& \text { I0.5 = " metallic silver, } \\
& 24.4=\text { " } \quad \text { platinum dish, } \\
& 8.5=" \text { weights, }
\end{aligned}
$$

and computed for the formula $\mathrm{AgC}_{2} \mathrm{H}_{8} \mathrm{O}_{2}$, assuming the atomic masses of carbon, hydrogen and oxygen to be $\mathrm{I} 2.0 \mathrm{I}, \mathrm{I} .008$ and I6, respectively, are as follows:

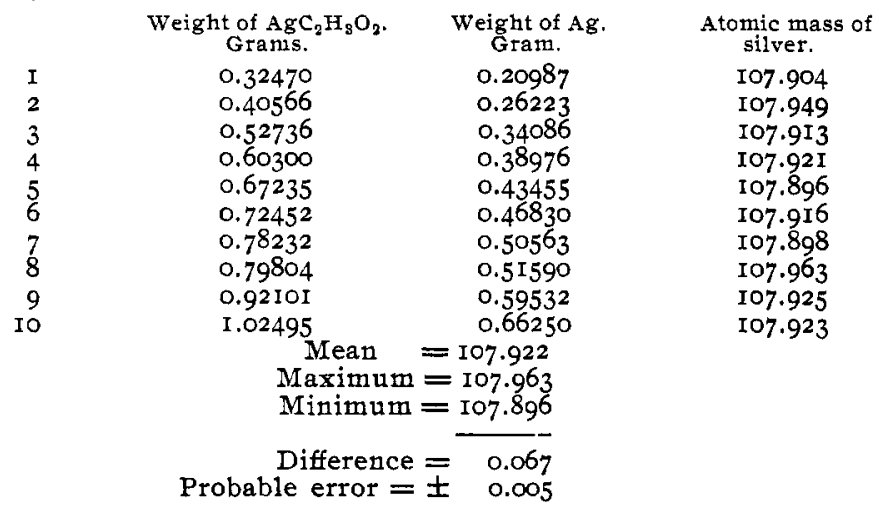

Computing from the total quantity of material used and metal obtained we have I07.91 8 for the atomic mass of silver. 


\section{EXPERIMENTS ON SILVER SLCCINATE.}

Silver succinate was prepared in a manner similar to that of silver acetate. The commercial C. P. succinic acid was recrystallized three times; the ammonium salt was then prepared and its aqueous solution precipitated with a solution of pure silver nitrates. The precipitate of silver succinate was thoroughly washed by decantation with pure water and carefully dried. After drying for several hours in an air-bath at $50^{\circ}$, the material was ground in an agate mortar to a finely divided powder, and was then redried for twenty-four hours in an air-bath at a temperature of $60^{\circ}$. The white powder obtained in this way was placed in a weighing tube and kept in a desiccator.

The method of analysis was similar to that of silver acetate. A weighed portion of the material was dissolved in a little potassium cyanide in a platinum dish. After diluting with pure water, the solution was electrolyzed and the resulting deposit weighed. The strength of current and time of action were the same as for silver nitrate. The results computed for the formula $\mathrm{C}_{4} \mathrm{H}_{4} \mathrm{O}_{4} \mathrm{Ag}_{2}$ were not constant, and were invariably from one to two units lower than those obtained from silver nitrate and silver acetate. The material was then dried at a temperature of $75^{\circ}$, but the results obtained were not satisfactory.

The two most probable causes for these low results are:

First, the difliculty of removing the last traces of impurities from a precipitate like that of silver succinate. The experience throughout this work has been, that, to remove all the impurities from a finely dirided precipitate by washing is almost impossible.

Second, the difficulty met in drying material of this kind. This same difficulty was met in the experiments on silver oxide which, as shown by Lea, retained moisture up to $165^{\circ}$.

\section{THIRD SERIES.}

EXPERIMENTS ON SILVER BENZOATE.

The preceding work on silver acetate and silver succinate shows the necessity of selecting compounds which form well defined crystals. Perhaps no organic salt of silver fulfils the 
ATOMIC MASSES OF SILVER, MERCURY AND CADMIUM. IOOI

conditions necessary for accurate analyses better than silver benzoate.

PREPARATION OF SILVER BENZOATE.

The purest commercial benzoic acid was resublimed three times from a porcelain dish into a glass beaker. The product thus obtained was dissolved in pure aqueous ammonia and the solution evaporated to crystallization. The ammonium salt was then dissolved in distilled water and to the solution was added a solution of pure silver nitrate. The white precipitate of silver benzoate which separated was washed with cold water; it was then dissolved in hot water, the solution filtered, and evaporated to crystallization. The salt separated in fine needles, which clung together in arborescent masses. After removing the liquid from the beaker, the crystals were quickly rinsed with cold water and placed between filters to remove the adhering moisture. When apparently dry they were broken up into small fragments and dried forty-eight hours in a vacuum desiccator. The material was then placed in a glass stoppered weighing tube and kept in a dark place.

MODE OF PROCEDURE.

The details of the method of operation are the same as those given under silver nitrate. A weighed portion of the material was dissolved in a dilute solution of potassium cyanide in a platinum dish. The solution was then electrolyzed and the resulting metal weighed. The strength of current and time of action were the same as for silver nitrate.

Before the results could be reduced to a vacuum standard it was necessary to determine the specific gravity of silver benzoate. This was done by means of a specific gravity bottle, the liquid used being chloroform. The mean of two determinations gave 2.082 for the specific gravity of silver benzoate.

Ten results on this compound, reduced to a vacuum standard on the basis of

$$
\begin{aligned}
& 2.082=\text { density of silver benzoate, } \\
& \text { I0.5 = " " metallic silver, } \\
& 2 \mathrm{I} .4=\text { " " " platinum dish, } \\
& 8.5=\text { " " weights, }
\end{aligned}
$$


and computed for the formula $\mathrm{C}_{1} \mathrm{H}_{5} \mathrm{AgO}_{2}$, assuming $\mathrm{I} 2.0 \mathrm{I}, \mathrm{I} .008$, and $I 6$ to be the atomic masses of carbon, hydrogen and oxygen, respectively, are as follows:

\begin{tabular}{|c|c|c|c|}
\hline & $\begin{array}{c}\text { Weight of } \mathrm{C}_{7} \mathrm{H}_{5} \mathrm{AgO}_{2} . \\
\text { Grams. }\end{array}$ & $\begin{array}{l}\text { Weight of Ag. } \\
\text { Gram. }\end{array}$ & $\begin{array}{l}\text { Atomic mass } \\
\text { of silver. }\end{array}$ \\
\hline I & $0.4085^{8}$ & 0.19255 & 107.947 \\
\hline 2 & 0.46674 & 0.21999 & 107.976 \\
\hline 3 & 0.48419 & $0.228 I_{5}$ & 107.918 \\
\hline 4 & 0.62432 & 0.29418 & IO 7.918 \\
\hline 5 & $0.6649^{6}$ & 0.31340 & 107.964 \\
\hline 6 & $0.75^{853}$ & 0.35745 & 107.935 \\
\hline 7 & 0.76918 & 0.36247 & 107.936 \\
\hline 8 & 0.81254 & 0.38286 & 107.914 \\
\hline 9 & 0.95673 & 0.45079 & 107.908 \\
\hline \multirow[t]{5}{*}{ IO } & 1.00840 & 0.47526 & 107.962 \\
\hline & Mean & $=107.938$ & \\
\hline & Maximum & $=107.976$ & \\
\hline & Minimum & $=107.908$ & \\
\hline & $\begin{array}{l}\text { Difference } \\
\text { Probable er }\end{array}$ & $\begin{array}{r}=0.068 \\
r= \pm 0.005\end{array}$ & \\
\hline
\end{tabular}

Computing from the total quantity of material used and metal obtained we have $107.93^{6}$ for the atomic mass of silver.

SUMMARY.

In discussing the work on the atomic mass of silver, two possible sources of error suggest themselves.

First, the hydrogen which is continually being set free in the process of electrolysis may, in part, be occluded by the metallic silver. As already pointed out, the metallic deposits were washed several times with boiling water, with the hope of removing any occluded gases; but whether this effected a complete removal of all the occluded gases was not determined.

Second, the condensation of moisture on the platinum dish might be urged as a possible source of error. But it must be remembered that the dish was dried in the same manner each time and kept for several hours in a desiccator, and that the atmosphere inside the balance was kept dry by means of several beakers of anhydrous calcium chloride, and that the temperature of the balance room throughout the work was almost constant. Under these conditions there is but little chance of error from 
different amounts of moisture condensed. Moreover, the variation in the different weighings of the same dish was very slight.

The advantages of the method are evident.

First, the great advantage of the method is its extreme simplicity.

Second, the nature of the compounds used and of metallic silver renders them well adapted to weighing.

Third, the method was such as to eliminate the errors incident to the ordinary gravimetric methods of analysis.

Of the three series, the first is probably entitled to the greatest weight. That the silver nitrate was pure and free from moisture seems beyond question. However, the close agreement of the last two series with the first indicates that the acetate and benzoate of silver were also free from moisture.

Giving equal weight to each of the three series, we have the following as the general mean computed from the separate observation :

Atonic mass of silver.

First series......................... I0 7.924

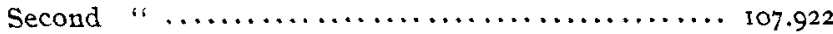

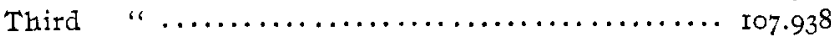

General mean $=107.928$

Computing the general mean from the total quantities of material used and metal obtained we have :

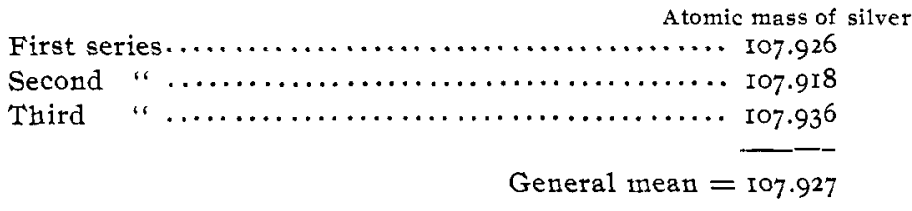

Combining this with the first general mean we have 107.9275 as the final result for the atomic mass of silver.

PART II.

DETERMINATION OF THE ATOMIC MASS OF MERCURY.

From all the earlier determinations Clarke gives 200 as the 
most probable value for the atomic mass of mercury, assuming oxygen equal to $I 6$.

\section{EXPERIMENTS ON MERCURIC OXIDE.}

A large number of experiments were made with a view of determining the ratio of mercury to oxygen in mercuric oxide. The method proved to be unsatisfactory, although, apparently very good results were obtained in some preliminary experiments. The cause of this close agreement of results will be explained in the details of the work.

PREPARATION OF PURE IIERCURIC OXIDE.

The purest commercial mercuric chloride was carefully sublimed from a porcelain dish into a glass funnel. The sublimed portion was dissolved in water, the solution filtered, and evaporated to crystallization. The crystals were then thoroughly dried and carefully resublimed. The product obtained in this way consisted of white crystalline leaflets which dissolved completely in water. Pure sodium hydroxide was then prepared by throwing pieces of metallic sodium on pure water contained in a platinum dish. To the pure sodium hydroxide was added a solution of mercuric chloride, the former always being in excess. The yellow mercuric oxide which separated was washed for several days by decantation with hot water. The material was then dried twenty-four hours in an air-bath at $105^{\circ}$.

MODE OF PROCEDURE.

In a series of preliminary experiments made in the spring of I 895 , a weighed portion of mercuric oxide prepared in the above manner was dissolved in a dilute solution of potassium cyanide in a platinum dish. The solution was then electrolyzed and the weight of the resulting metallic mercury determined. Inasmuch as the results obtained in these preliminary experiments were not reduced to a vacuum standard, it was thought advisable to weigh the empty platinum dish after removing the metallic deposit in order that the two weighings might be made under approximately the same conditions. The results for the most part agreed very closely and differed very little from the results obtained by other methods. Six observations computed for the formula $\mathrm{HgO}$, assuming the atomic mass of oxygen to be 16 , are as follows: 


$\begin{array}{cccc} & \begin{array}{c}\text { Weight of HgO. } \\ \text { Gram. }\end{array} & \begin{array}{c}\text { Weight of } \mathrm{Hg} . \\ \text { Gram. }\end{array} & \begin{array}{c}\text { Atomic mass } \\ \text { of metcury. }\end{array} \\ \text { I } & 0.26223 & 0.2428 \mathrm{I} & 200.05 \\ 2 & 0.23830 & 0.22065 & 200.02 \\ 3 & 0.23200 & 0.21482 & 200.06 \\ 4 & 0.14148 & 0.13 \mathrm{I} 100 & 200.00 \\ 5 & 0.29799 & 0.27592 & 200.03 \\ 6 & 0.1963 \mathrm{I} & 0.18 \mathrm{I} 77 & 200.02\end{array}$

These results were selected from a larger series. After making the above observations it was noticed that the platinum dish had gradually decreased in weight throughout the work. This decrease in weight indicated that the mercury deposit had formed an amalgam with the platinum dish, which was soluble in hot nitric acid. To ascertain whether such was the case or not the platinum dish, after weighing was filled with a solution of the double cyanide of mercury and potassium and the solution electrolyzed. On dissolving the mercury deposit in cold nitric acid a dark colored film remained on the sides of the dish. The dish was then carefully washed, dried and reweighed, and found to be heavier than at the beginning of the operation, showing that the mercury had not been completely removed. The dark film was then dissolved in hot nitric acid and the dish again weighed. This last weight being less than that at the beginning showed that some of the platinum had been dissolved from the dish. The nitric acid solution of the dark film was evaporated to dryness and ignited to remove the mercury. The residue was dissolved in aqua regia, the solution evaporated to dryness, and enough water added to dissolve the small residue. A little concentrated ammonium chloride was then added to the solution, and the double chloride of ammonium and platinum separated as a yellow crystalline powder. This proved conclusively that the mercury deposit had united with the platinum dish to form an amalgam which was soluble in hot nitric acid. Hence the results given for mercuric oxide are of no value in determining the atomic mass of mercury.

A series of careful experiments was then made on the oxide dried at different temperatures. To avoid any error from the 
amalgam which formed with each deposit, the platinum dish was weighed at the beginning of each observation, the temperature and barometric pressure being noted at the same time. The results obtained from the oxide dried at a temperature of $105^{\circ}$ gave from 180 to 185 for the atomic mass of mercury. The material was then dried at a temperature of $125^{\circ}$, but the increase in the amount of mercury obtained was very slight. Finally with material dried at $150^{\circ}$, the results obtained for the atomic mass of mercury were all below $195^{\circ}$.

The most probable causes for these low results are:

First, the difficulty of removing the last traces of alkalies from the mercuric oxide.

Second, the difficulty met in the complete removal of the moisture from an amorphous precipitate. This difficulty as well as the first was referred to in the experiments on silver oxide.

Third, mercuric oxide does not form a clear solution with potassium cyanide. There seems to be a slight reduction of the oxide to the metallic state. It is difficult to determine whether this reduced portion unites completely with the metallic deposit or is partially removed in the process of washing. The latter is probably true, and it may be that a different method of analysis would give more accurate results for this compound.

\section{FIRST SERIES.}

EXPERIMENTS ON MERCURIC CHLORIDE.

The material used in this series of experiments was prepared from the commercial C. P. mercuric chloride. The product was first dissolved in water, the solution filtered and evaporated to crystallization. The crystals were dried and carefully sublined from a porcelain dish into a glass funnel. The sublimed portion was dissolved in water, the solution filtered and evaporated to crystallization. These crystals were dried as before and carefully resublimed. The material was then placed in a weighing tube and kept in a desiccator.

MODE OF PROCEDLRE.

The method of operation was similar to that already described 
under the different compounds of silver. A weighed portion of the mercuric chloride was dissolved in a little potassium cyanide and the solution electrolyzed. The deposit was washed and dried and handled in every way like the deposits of silver. The strength of the current and time of action were as follows :

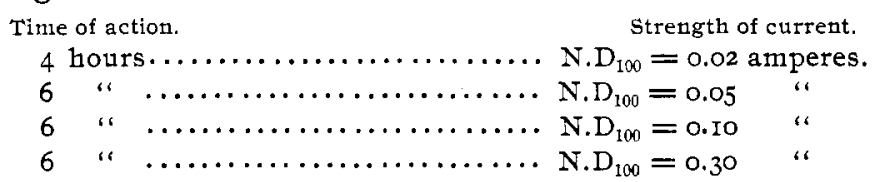

A current of gradually increasing strength deposits the mercury in extremely small globules, which can be washed and handled more easily than the larger globules obtained by using a strong current at first. In cases where more than one-half gram of metal was deposited the strong current was allowed to act two hours longer.

Ten results on mercuric chloride reduced to a vacuum standard on the basis of

$$
\begin{aligned}
& 5.4 \mathrm{I}=\text { density of mercuric chloride, } \\
& \mathrm{r} 3.59==\text { " metallic mercury, } \\
& 2 \mathrm{I} .4= \text { " }=\text { " } \\
& 8.5=\text { weightinum dish, }
\end{aligned}
$$

\begin{tabular}{|c|c|c|c|}
\hline & $\begin{array}{c}\text { Weight of } \mathrm{HgCl}_{2} \text {. } \\
\text { Grams. }\end{array}$ & $\begin{array}{l}\text { Weight of Hg. } \\
\text { Grams. }\end{array}$ & $\begin{array}{l}\text { Atomic mass of } \\
\text { mercury. }\end{array}$ \\
\hline $\mathbf{I}$ & 0.45932 & 0.33912 & 200.030 \\
\hline 2 & 0.54735 & 0.40415 & 200.099 \\
\hline 3 & 0.56002 & $0.4 J \cdot 348$ & 200.053 \\
\hline 4 & $0.635^{86}$ & $0.4694 \mathrm{I}$ & I99.947 \\
\hline 5 & $0.643^{65}$ & $0.475^{21}$ & 200.026 \\
\hline 6 & $0.7328 \mathrm{I}$ & 0.54101 & I 99.988 \\
\hline 7 & 0.86467 & 0.63840 & 200.838 \\
\hline 8 & I. .06776 & 0.78825 & 199.946 \\
\hline 9 & I. . 7945 & 0.79685 & 199.917 \\
\hline IO & I.5I 402 & I.II 780 & 200.028 \\
\hline \multicolumn{4}{|c|}{ Mean $=200.006$} \\
\hline \multicolumn{4}{|c|}{ Maximum $=200.099$} \\
\hline \multicolumn{4}{|c|}{ Minimum $=199.9 \mathrm{I} 7$} \\
\hline & Differ & $=0.182$ & \\
\hline & Probable e & $= \pm 0.0 \mathrm{II}$ & \\
\hline
\end{tabular}

and computed from the formula $\mathrm{HgCl}_{2}$, assuming 35.45 to be the atomic mass of chlorine, are as follows: 
Computing from the total quantity of material used and metal obtained we have 199.996 for the atomic mass of mercury.

SECOND SERIES.

\section{EXPERIMENTS ON MERCURIC BROMIDE.}

The bromine used in these experiments was prepared by distilling the commercial C. P. bromine twice over manganese dioxide. Any trace of chlorine which might be present would be removed by this method.

PREPARATION OF MERCURIC BROMIDE.

Fifty grams of metallic mercury were placed in a beaker and covered with water. Pure bromine was then added until the mercury was completely saturated. The contents of the beaker were then digested with hot water until the mercuric bromide dissolved; the solution was filtered and evaporated to crystallization. The white crystals of mercuric bromide which separated were thoroughly dried and carefully sublimed from a porcelain dish into a glass funnel. Only the middle portion of the sublimate was used in the experiments. The product obtained in this way consisted of brilliant crystalline leaflets which dissolved completely in water. The material was kept in a weighing tube in a desiccator.

MODE OF PROCEDURE.

The method of analysis was exactly like that described under mercuric chloride. A weighed portion of the mercuric bromide was dissolved in dilute potassium cyanide in a platinum dish. The solution was then electrolyzed and the resulting metal weighed. The strength of current and time of action were the same as for mercuric chloride.

Ten results on mercuric bromide reduced to a vacuum standard on the basis of

$$
\begin{aligned}
& 5.92=\text { density of mercuric bromide, } \\
& \text { I } 3.59=\text { " " metallic mercury, } \\
& 2 \mathrm{r} .4=\text { " " " watinum dish, } \\
& 8.5=\text { "weights, }
\end{aligned}
$$


and computed for the formula $\mathrm{HgBr}_{2}$, assuming 79.95 to be the atomic mass of bromine, are as follows :

\begin{tabular}{|c|c|c|c|}
\hline & $\begin{array}{l}\text { Weight of } \mathrm{HgBr}_{2} \\
\text { Grams. }\end{array}$ & $\begin{array}{l}\text { Weight of } \mathbf{H g} . \\
\text { Grams. }\end{array}$ & $\begin{array}{l}\text { Atomic mass of } \\
\text { mercury. }\end{array}$ \\
\hline I & 0.70002 & 0.38892 & 199.898 \\
\hline 2 & 0.56430 & 0.31350 & 199.876 \\
\hline 3 & 0.57142 & $0.3175^{\circ}$ & $199.93^{8}$ \\
\hline 4 & $0.77^{285}$ & 0.42932 & 199.832 \\
\hline 5 & 0.80930 & 0.44955 & $199.8 \mathrm{I} 4$ \\
\hline 6 & 0.85342 & 0.47416 & I99.9II \\
\hline 7 & I. 11076 & 0.6 I 708 & 199.869 \\
\hline 8 & 1.17270 & 0.65 I 45 & 199.840 \\
\hline 9 & I. 26 I 86 & 0.70107 & I 99.899 \\
\hline 10 & I. 40142 & $0.77^{870}$ & I 99.952 \\
\hline & & $=199.88_{3}$ & \\
\hline \multirow{2}{*}{\multicolumn{4}{|c|}{$\begin{aligned} \text { Maximum } & =199.952 \\
\text { Minimum } & =199.8 \mathrm{I} 4\end{aligned}$}} \\
\hline & & & \\
\hline & Differe & $=0.138$ & \\
\hline & Probable e & $= \pm 0.010$ & \\
\hline
\end{tabular}

Computing from the total quantity of material used and metal obtained, the atomic mass of mercury is 199.885 .

\section{THIRD SERIES.}

\section{EXPERIMENTS ON MERCURIC CYANIDE.}

A series of observations was made on several organic salts of mercury with a view of selecting a compound suitable for atomic mass determinations. Mercuric acetate and other similar salts were found to be unstable in the air and unsuited for accurate analyses. Mercuric cyanide, on the other hand, was found to be perfectly stable and to form well defined crystals.

$$
\text { PREPARATION OF HYDROCYANIC ACID. }
$$

Five hundred grams of potassium ferrocyanide were placed in a two liter retort connected with a condenser. A cooled mixture of 300 grams of pure sulphuric acid and $700 \mathrm{cc}$. of distilled water was then poured into the retort and the mixture carefully heated until the hydrocyanic acid was distilled over into the receiver. The product obtained was redistilled and used immediately in the preparation of mercuric cyanide. 


\section{PREPARATION OF MERCURIC CYANIDE.}

Fifty grams of mercuric oxide, prepared as already described in the experiments on mercuric oxide, were dissolved in pure, warm hydrocyanic acid. The solution was then filtered and evaporated to crystallization. The transparent crystals of mercuric cyanide which separated were dissolved in pure water and recrystallized. The product obtained by the second crystallization was quickly rinsed with cold water and dried for six hours in an air bath at a temperature of $50^{\circ}$. The crystals were then ground to a finely divided powder in an agate mortar and redried for twenty-four hours in an air bath at a temperature of $55^{\circ}$. The dry, white powder was then placed in a weighing tube and kept in a desiccator.

\section{MODE OF PROCEDURE.}

The mode of procedure with mercuric cyanide was somewhat different from that of the preceding experiments, in that no potassium cyanide was used in preparing the solution for electrolysis. A weighed portion of the material was dissolved in pure water in a platinum dish. When the crystals had completely dissolved, the dish was filled to within a quarter of an inch of the top with water, after which one drop of pure sulphuric acid was added. The solution was then electrolyzed and the resulting metal weighed. The strength of the current and the time of action were the same as for mercuric chloride. In the last four experiments, where rather large quantities of mercury were deposited, the strong current was allowed to act from two to six hours longer.

The results of ten experiments on mercuric cyanide, reduced to a vacuum standard on the basis of

$$
\begin{aligned}
4.0 & =\text { density of mercuric cyanide, } \\
\mathrm{r} 3.59 & =" \text { " " } \\
2 \mathrm{I} .4 & =\text { " } \\
8.5 & =\text { " }
\end{aligned}
$$

and computed for the formula $\mathrm{Hg}(\mathrm{CN})_{2}$, assuming $\mathrm{I} 2 . \mathrm{OI}$ and I 4.04 to be the atomic masses of carbon and nitrogen, respectively, are as follows : 


$\begin{array}{rccc}\text { Weight of } \mathrm{Hg}(\mathrm{CN})_{2} . & \text { Weight of } \mathrm{Hg} . & \begin{array}{c}\text { Atomic mass } \\ \text { of mercury. } \\ \text { Grams. } \\ \text { Grams. }\end{array} \\ 2 & 0.55776 & 0.44252 & 200.063 \\ 3 & 0.63290 & 0.50215 & 200.092 \\ 4 & 0.70652 & 0.56053 & 200.038 \\ 5 & 0.8024 \mathrm{I} & 0.63663 & 200.075 \\ 6 & 0.65706 & 0.52130 & 200.057 \\ 7 & 0.81678 & 0.64805 & 200.103 \\ 8 & 1.07628 & 0.85392 & 200.077 \\ 9 & 1.22615 & 0.97282 & 200.07 \mathrm{I} \\ 10 & 1.66225 & 1.31880 & 200.057 \\ & 2.11 \mathrm{I} 7 \mathrm{O} & \mathrm{1} .6754 \mathrm{I} & 200.077 \\ & \text { Mean } & =200.07 \mathrm{I} & \\ & \text { Maximum } & =200.103 & \\ & \text { Minimum } & =200.038 & \\ & \text { Difference } & =0.065 & \\ & \text { Probable error } & =0.005\end{array}$

From the total quantity of material used and metal obtained, the atomic mass of mercury is 200.070 .

\section{FOURTH SERIES.}

According to Faraday's law the quantities of different metals deposited from their solutions by the same current are proportional to their equivalent weights. In this series of experiments an attempt was made to determine the ratio of the atomic mass of mercury to that of silver by passing the same current through the solutions of the two metals and weighing the two resulting deposits. If the proper conditions could be obtained, this would certainly be the simplest and most direct method for comparing the equivalent weights of different metals. But so many difficulties were met that the method on the whole was not satisfactory.

In the "Revision of the Atomic Weight of Gold," Mallet made use of this method, and in a series of careful preliminary experiments determined the conditions most favorable to its application. From a number of experiments made by passing the same current through two different solutions of copper sul-

1 Am. Chem.J., 12, 182. 
phate, using pure electrotype copper for both anode and cathode in each solution, Mallet found:

First.-Other conditions being the same, the difference in the quantities of metal deposited from solutions of unequal concentrations was very slight and somewhat variable, but the tendency was toward a slightly larger quantity from the more concentrated solution.

Second.-With equal quantities of metal in the two solutions and unequal quantities of free acid, the difference in the results obtained were almost insignificant and somewhat variable in direction, the tendency being toward a slightly larger quantity from the less acid solution.

Third.-Other conditions being the same, a difference in the temperature of the two solutions invariably caused a slightly larger deposit from the cooler solution.

Fourth.-Other conditions being the same, a difference in the size of the copper plates, and hence a difference in the "current density," caused a slightly greater deposit on the smaller plate.

Fifth.-A difference in the distance between the two plates did not produce a constant difference of result, but the tendency was toward a slightly larger deposit on the cathode plate farther separated from its anode.

From the foregoing experiments it is evident that the conditions most favorable to this method are, that the two solutions should be equally concentrated, of the same temperature, and should contain equal amounts of free acid, or when the double cyanides are used, equal quantities of free potassium cyanide. And, moreover, that the two cathodes and also the two anodes should be of the same size, and that the distance between the anode and cathode should be the same in both solutions. These conditions were closely observed throughout this work.

ARRANGEMENT OF APPARATUS.

The deposits in this series of experiments were made in two platinum dishes of equal capacity and equal internal area. The anode in each case consisted of a coil of rather large platinum wire, the two coils being of the same shape and size. The dishes were insulated from each other by means of two glass stands. 
The platinum coils were completely immersed in the solutions and the portion of the wire near the surface of the liquid was covered with paraffin to prevent surface contact. The surrent, after passing through the two solutions, was allowed to pass through a hydrogen voltameter in order that its strength might be observed at any time.

In the second arrangement of apparatus the platinum dishes were made the anodes, and two pieces of platinum foil of the same shape and size were used for the cathodes. The results, however, from this second arrangement were not as satisfactory as from the first.

\section{MODE OF PROCEDURE.}

A solution of the double cyanide of siiver and potassium was placed in one of the platinum dishes and a solution of the double cyanide of mercury and potassium in the other. The quantities of silver and mercury present in their solutions were approximately proportional to their equivalent weights. Each solution contained a slight excess of potassium cyanide. The dishes were placed in their positions and the anodes immersed sometime before the current was allowed to act. When the temperature of the two solutions was the same as that of the room, the connection was made and the same current allowed to pass through the two solutions. The quantity of metal deposited was never allowed to exceed one-half of the metal present in the solution at first. Before interrupting the current, the solutions were siphoned from the two platinum dishes at the same time with two siphons of the same bore. The deposits were then washed several times with boiling water, carefully dried and their weights determined. Experiments were made with currents of different strength and with solutions of varions degrees of concentration. The results obtained were far from being satisfactory. The strength of current which seemed best adapted to the work was that which deposited about one-tenth of a gram of silver per hour.

From a large number of experiments, only seven results were obtained which seem of any value in determining the atomic mass of mercury. And it must be added that many others were 
rejected, not because they were known to be vitiated in any way, but because the results obtained for the atomic mass of mercury differed from those obtained by other methods. It is possible that, in a large number of experiments, the condition would be more favorable in some than in others, but whether the close agreement of the results selected was due to this or to the balancing of errors, could not be determined.

Seven results computed on the basis of 107.92 for the atomic mass of silver are as follows:

\begin{tabular}{|c|c|c|c|}
\hline & $\begin{array}{l}\text { Weight of } \mathrm{Hg} \text {. } \\
\text { Gram. }\end{array}$ & $\begin{array}{l}\text { Weight of } \mathrm{Ag} \text {. } \\
\text { Gram. }\end{array}$ & $\begin{array}{l}\text { Atonic mass } \\
\text { of mercury. }\end{array}$ \\
\hline I & 0.016126 & 0.06610 & 200.036 \\
\hline 2 & 0.06190 & 0.06680 & 200.007 \\
\hline 3 & $0.078 \mathrm{I} 4$ & 0.08432 & $200.02 I$ \\
\hline 4 & 0.10361 & $0 . \operatorname{III} 8 \mathrm{I}$ & $200.0 \mathrm{II}$ \\
\hline 5 & $0.1520 I$ & 0.16 .402 & $200.06 I$ \\
\hline 6 & 0.26806 & 0,28940 & I99.924 \\
\hline \multirow[t]{5}{*}{7} & 0.82808 & 0.89388 & 199.929 \\
\hline & \multicolumn{2}{|r|}{$=199.996$} & \\
\hline & \multicolumn{2}{|c|}{ Maximum $=200.06 \mathrm{I}$} & \\
\hline & \multicolumn{2}{|c|}{ Minimum $=199.924$} & \\
\hline & \multicolumn{2}{|c|}{ Difference $=\overline{0.137}$} & \\
\hline
\end{tabular}

Computing from the total quantities of mercury and silver obtained, we have 199.97 I for the atomic mass of mercury.

Although the cause of the large variation in the rejected observations could not be definitely determined, several sources of error suggest themselves.

First, small quantities of hydrogen were undoubtedly set free in the process of electrolysis, and unless these quantities were always equal in the two solutions, which is not probable, an error would be introduced.

Second, in some solutions an error might easily be introduced by a change in the atomicity of mercury, but in a solution of the double cyanide of mercury and potassium this change is hardly probable.

Third, the occlusion of hydrogen by the two metallic deposits would also be a possible source of error; but only small errors could be introduced in this way.

To account for the difference of several units in the results, 
the source of error first mentioned seems by far the most probable.

\section{SUMMARY.}

In the discussion of the results obtained in the different series of observations on the compounds of silver, the probable sources of error and likewise the advantages of the method were pointed out. The same discussion applies equally well to the observations on mercury.

It is evident that the first three series of observations on mercury are entitled to more weight than the last series. Just why the results on mercuric bromide should be lower than those on mercuric chloride is not clear. Both compounds are certainly well adapted to atomic mass determinations, inasmuch as they can be purified by both crystallization and sublimation. The most probable impurity in mercuric bromine would be mercuric chloride, but that would tend to increase rather than lower the results. The series of observations on mercuric cyanide have, perhaps, one advantage over the others, in that no potassium cyanide was used. The results obtained in this series are still bigher than those obtained from mercuric chloride and almost two-tenths of a unit higher than those obtained from mercuric bromide. However, as the same care was exercised in the purification of the material for each of the three series, and as there was no apparent error in either case, equal weight must be given to each of the three series in determining the most probable value of the atomic mass of mercury. And, as the mean of the last series is almost identical with the mean of the first three, equal weight can be given to this series without introducing any error.

Computing the general mean from the separate observations, we have:

Atomic mass of mercury.

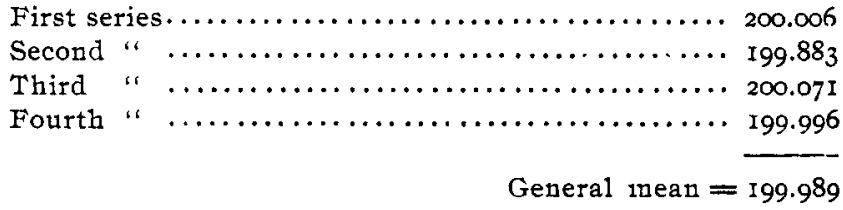


From the total quantities of material used and metal obtained, the general mean is :

Atomic nuass of mercury.

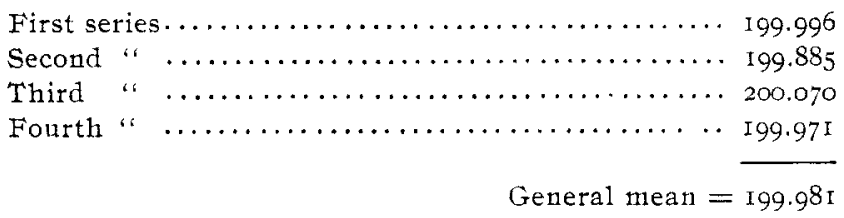

Combining this with the first general mean we have:

Atomic mass of mercury.

First general mean $=199.989$

Second " " $=199.98 \mathrm{I}$

Most probable mean of all the results $=199.985$

or 200 for the atomic mass of mercury.

\section{PART III.}

DETERMINATION OF THE ATOMIC MASS OF CADMIUM.

Nine experimenters have determined the atomic mass of cadmium by many different methods, but the large variations in the results given by different chemists leave the true value of this constant still uncertain.

Stromeyer ${ }^{1}$ gave no details of his method of operation, but found that 100 parts of cadmium combined with 14,352 parts of oxygen. On the basis of $O=I 6$, this ratio gives III.483 for the atomic mass of cadmium. This result is much lower than those obtained by other experimenters and is perhaps only of historical interest.

In a series of nine experiments, Von Hauer ${ }^{2}$ determined the ratio of cadmium sulphate to cadmium sulphide. The sulphate used was purified by repeated recrystallizations and was finally dried at a temperature of $200^{\circ}$. After weighing the sulphate was always dried a second time and reweighed. The two weighings never differed as much as one milligram. The sulphide obtained was in each case tested for sulphate. The reduction of the sulphate to sulphide was accomplished by heating

1 Berzelius' Lehrbuch, 5 th Ed., 3, I2r9

2 J.prakt. Chem., 72, 350 . 
the sulphate in a current of dry hydrogen sulphide under pressure. The mean of nine observations computed on the basis ) $\mathrm{O}=\mathrm{I} 6$ and $\mathrm{S}=32.06$ gives III.93 for the atomic mass of cadmium. Considering the large quantity of material used each time and the precautions taken to insure accuracy, there seems to be little objection to the method.

Dumas ${ }^{1}$ determined the ratio of cadmium chloride to metallic silver by titrating a solution containing a weighed quantity of cadmium chloride with a silver nitrate solution of known strength. The cadmium chloride was prepared by dissolving metallic cadmium in boiling hydrochloric acid. The solution was evaporated to dryness and the chloride fused for six hours in a current of hydrochloric acid gas. The mean of six determinations gives I 2.24 for the atomic mass of cadmium $(\mathrm{O}=16)$.

$$
\begin{aligned}
\text { Maximum result, } \mathrm{Cd} & =\operatorname{II2.759} \\
\text { Minimum " } \mathrm{Cd} & =\operatorname{III.756} \\
\text { Difference } & =-\mathrm{I.003}
\end{aligned}
$$

This large variation in the results obtained indicates the presence of impurities in the material used. In the first three experiments the cadmium was not purified; the mean of these three is $\mathrm{Cd}=\mathrm{II} 2.476$. The metal used in the last three experiments was considered by Dumas to be absolutely pure; the mean of the last three results is $\mathrm{Cd}=\mathrm{II} 2.007$. From the degree of purity of the cadmium chloride used in the different experiments, Dumas was inclined to reject the higher results and concluded that the true atomic mass of cadmium was about I I 2 .

Lensen ${ }^{2}$ prepared pure cadmium oxalate by precipitating a solution of cadmium chloride, purified by repeated crystallization, with pure oxalic acid. The precipitate was washed and carefully dried at a temperature of $150^{\circ}$. The mean of three results obtained by converting a weighed portion of the oxalate to oxide gives I 12.06 for the atomic mass of cadmium $(\mathrm{O}=\mathrm{I} 6)$. The small quantity of material used in the different experiments is somewhat objectionable.

1 Ann. chim. phys., [3], 55, 158.

2 J.prakt. Chem., 79, $28 \mathrm{I}$. 
Huntington, ${ }^{1}$ under the direction of Cooke, determined the ratio of cadmium bromide to silver bromide and also the ratio of cadmium bromide to metallic silver. The bromide used was prepared by dissolving cadmium carbonate, which had been carefully purified, in pure hydrobromic acid. The product obtained was dried at a temperature of $200^{\circ}$ and finally sublimed in a porcelain tube in a current of dry carbon dioxide. In the first series of experiments the silver bromide corresponding to the cadmium bromide used. was weighed. The mean of eight determinations computer from the total quantity of material used and silver bromide obtained, on the basis of $\mathrm{Ag}=107.93$ and $\mathrm{Br}=79.95$ is $\mathrm{Cd}=\mathrm{I} 2.24$. In the second series of experiments the quantity of metallic silver required to precipitate a known quantity of cadmium bromide was determined. The mean of eight determinations computed as in the first series gives $1 \times 2.245$ for the atomic mass of cadminm. The separate determinations in both series agree very closely.

Partridge made three series of determinations. The first depended upon the conversion of cadmium oxalate into oxide, the second, on the reduction of the sulphate to sulphide, and the third, on the conversion of the oxalate into sulphide. The cadmium used in these experiments was purified by distilling twice in vacuo. Tell observations on the conversion of the oxalate into oxide, computed on the basis of $\mathrm{O}=16$ and $\mathrm{C}=12$, give III.80I as a mean for the atomic mass of cadmium. Recalculated by Clarke, ${ }^{3}$ on the basis of $\mathrm{O}=\mathrm{I} 6$ and $\mathrm{C}=12.005$, the atomic mass of cadnium becomes IIr.818. The mean of ten results obtained by reducing the sulphate to sulphide, computed on the basis of $O=I 6$ and $S=32$, gives III.797 for the atomic mass of cadmium. Recalculated by Clarke on the basis of $\mathrm{O}=$ I 6 and $S=32.074$, the atomic mass of cadmium is III.7II. In the third series the oxalate of cadmium was converted into sulphide by heating in a current of dry hydrogen sulphide. The mean of ten determinations, computed on the basis of $O=I 6$ and $S=32$, gives III.805 for the atomic mass of cadmium.

\footnotetext{
1 Proc. Amer. Acad., I7, 28.

2 Am. J. Sci., [3], 40, 37.

3 Am. Chem. J., I3. 34 .
} 
Recalculated by Clarke on the basis of $\mathrm{O}=16$ and $\mathrm{S}=32.074$, the mean becomes III.589. Partridge gives III. 8 for the atomic mass of cadmium, as a mean of the three series. If the higher values for carbon and sulphur be introduced this value becomes somewhat lower.

Jones ${ }^{1}$ determined the atomic mass of cadmium by two different methods. The first was based on the conversion of the metal into oxide, and the second on the conversion of the oxalate into oxide. The cadmium used was distilled six times in vacuo. The last distillate was tested spectroscopically and found to be free from impurities. In the first series of experiments a weighed portion of the pure metal was dissolved in pure nitric acid in a porcelain crucible. The solution was evaporated to dryness and the resulting cadmium nitrate ignited to oxide. The final decomposition was accomplished by means of a blast lamp. Reducing gases were carefully excluded from the crucible during the process of ignition. The weighings were all made against a tared crucible. The mean of ten observations, computed on a basis of $O=I 6$ gives $I 12.07$ for the atomic mass of cadmium. The different determinations agree very closely. In the second series of experiments cadmium oxalate, prepared by precipitating pure cadmium nitrate with pure oxalic acid, was converted into oxide. The material was carefully ignited until the oxalate was decomposed; it was then treated with nitric acid and again ignited in a manner similar to that described in the first series. The mean of five determinations computed on the basis of $\mathrm{O}=\mathrm{I} 6$ and $\mathrm{C}=\mathrm{I} 2.003$ is $\mathrm{Cd}=\mathrm{III.032}$. From all the observations, Jones concludes that 1 I 2.07 represents very closely the atomic mass of cadmium $(\mathrm{O}=16)$.

Lorimer and Smith ${ }^{2}$ determined the ratio of the atomic mass of cadmium to that of oxygen by dissolving pure cadmium oxide in potassium cyanide and electrolyzing the solution. To obtain pure material, the commercial cadmium was dissolved in nitric acid and the solution evaporated to crystallization. The crystals of cadmium nitrate were removed from the liquid, dissolved in pure water and recrystallized. The product obtained by the

$1 \mathrm{Am}$. Chem. J., 14, 26r.

2 Ztschr. anorg. Chem., s, 364 . 
second recrystallization was dissolved in a little water and treated with a slight excess of potassium cyanide in a platinum dish. From this solution the metallic cadmium was thrown out by means of the electric current. The nitrate obtained by dissolving the electrolytic cadmium in pure nitric acid was tested spectroscopically and found to be free from impurities. The pure cadmium nitrate was digested with ammonium hydroxide and ammonium carbonate and the resulting cadmium carbonate ignited to oxide in a platinum crucible. The method of operation was very simple, a weighed portion of the oxide was dissolved in pure potassium cyanide, the solution electrolyzed and the resulting metallic cadmium weighed. The mean of nine observations computed on the basis of $O=$ r 6 gives II 2.055 for the atomic mass of cadmium.

Bucher ${ }^{1}$ made six series of experiments. The cadmium used was purified by nine distillations in vacuo. The weighings were all reduced to a vacuum standard and computed on the basis of $\mathrm{O}=16, \mathrm{~S}=32.059, \mathrm{C}=12.003, \mathrm{Cl}=35.45, \mathrm{Br}=79.95$, and $\mathrm{Ag}=107.93$.

In the first series cadmium oxalate, dried for fifty hours at $150^{\circ}$, was ignited to oxide. The mean of eight observations gives I I .89 for the atomic mass of cadmium.

In the second series, cadmium oxalate was converted into sulphide by heating in a current of dry hydrogen sulphide. The mean of four determinations is $\mathrm{Cd}=$ II2.I5.

In the third series a weighed quantity of cadmium chloride. dried at a temperature of $300^{\circ}$ in hydrochloric acid gas, was precipitated with silver nitrate and the resulting silver chloride weighed. The mean of twenty-one determinations is $\mathrm{Cd}=\mathrm{I} 12.39$. The separate observations in this series agree very closely.

The fourth series was similar to the third, except that cadmium bromide was used instead of the chloride. The mean of five determinations is $\mathrm{Cd}=\mathrm{I} 12.38$, a result almost identical with that obtained from the chloride.

In the fifth series a weighed portion of metallic cadmium was converted into sulphate, which was dried at $400^{\circ}$ and weighed.

1 Thesis, Johns Hopkins University, 2894 . 
The excess of sulphuric acid which remained with the sulphate was estimated and its weight deducted. The only result given is $\mathrm{Cd}=\mathrm{I} 12.35$.

In the last series metallic cadmium was converted into oxide by dissolving in nitric acid and igniting the resulting cadmium nitrate. The mean of two determinations made by igniting the material in a porcelain crucible gives I 2.08 for the atomic mass of cadmium. Three similar determinations made with a platinum crucible gave as a mean $\mathrm{Cd}=\mathrm{Ir}$.87. From a series of experiments on cadmium oxide, Bucher concluded that a correction should be applied to the last and also the first series. By making this correction, the results in these two series would be very close to those obtained from the chloride and bromide.

From all the preceding determinations Clarke gives III.93 as the most probable value for the atomic mass of cadmium. The large variation in the results of different experimenters has not been fully explained. Some chemists think that the larger values are due to a higher degree of purity in the metallic cadmium used, and hence regard these values as being more nearly correct. But it must be remembered that the reverse is true in the experiments of Dumas. From material which had not been purified, Dumas obtained results ranging from I 2.32 to I 12.76 for the atomic mass of cadmium, while from material which he considered absolutely pure, the results were from III.76 to I I 2. I 3 .

\section{PREPARATION OF PURE CADMIUM.}

The metallic cadmium used in these experiments was purified by distillation in a current of hydrogen which had been passed through solutions of caustic potash, lead nitrate, potassium permanganate, and sulphuric acid. A hard glass combustion tube was heated to redness and the walls of the tube indented at two points with a three-cornered file. This divided the tube into three parts. Commercial cadmium was placed in one end of the tube and connection made with the hydrogen generator. After complete removal of the air, the tube was carefully heated in a combustion furnace until one-half of the metal had distilled over into the middle portion of the tube. The metal was cooled in a 
current of hydrogen. The tube was then broken and the metal removed. The portions in the first and last sections of the tube were rejected. The middle portion was placed in a second combustion tube, similar to the first, and the distillation repeated. After three distillations the metal was examined spectroscopically and found to be free from impurities.

\section{FIRST SERIES. \\ EXPERIMENTS ON CADIIIUA CHLORIDE.}

Dumas and Bucher have both determined the ratio of cadmium to chlorine in cadmium chloride. The results given for the atomic mass of cadmium by the latter experimenter are almost four-tenths of a unit higher than those given by the former.

\section{PREPARATION OF CADMIUM CHLORIDE.}

Hydrochloric acid was purified by first passing chlorine through the commercial C. P. acid to remove any sulphur dioxide; the excess of chlorine was removed by a current of carbon dioxide. The acid was then distilled from calcium chloride and the hydrochloric acid gas collected in pure water. Pure metallic cadmium was then dissolved in the acid and the solution evaporated to crystallization. The crystals of cadmium chloride were removed from the liquid and thoroughly dried. The material was then placed in a hard glass combustion tube. similar to that used in the distillation of metallic cadmium, and carefully sublimed in a current of dry carbon dioxide. The first and last portions of the sublimate were rejected. The middle portion, which consisted of pearly leaflets, was placed in a weighing tube and kept in a desiccator. As only a small quantity of the material could be sublimed at a time, the different analyses were made from different sublimations.

MODE OF PROCEDURE.

A weighed portion of the cadmium chloride was dissolved in a little water in a platinum dish. A slight excess of potassium cyanide was added and, after diluting to $200 \mathrm{cc}$. with pure water, the solution was electrolyzed. Before interrupting the current, the liquid was siphoned from a dish in a manner already outlined 
ATOMIC MASSES OF SILVER, MERCURY AND CADMIUM. IO23

in the experiments on silver. The metallic deposit was washed several times with boiling water and carefully dried. The strength of the current and time of action were as follows:

Time of action. strength of current.

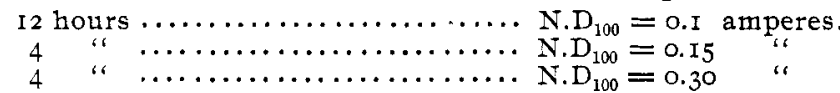

The cadmium was thrown down as a dense white deposit.

Ten results on cadmium chloride reduced to a vacuum standard on the basis of :

$$
\begin{aligned}
3.3 & =\text { density of cadmium chloride, } \\
8.55 & =\text { " " metallic cadmium, } \\
21.4 & =\text { " "platinum dish, } \\
8.5 & =\text { " "weights, }
\end{aligned}
$$

\begin{tabular}{|c|c|c|c|}
\hline & $\begin{array}{l}\text { Weight of } \mathrm{CdCl}_{2} \text {. } \\
\text { Grams. }\end{array}$ & $\begin{array}{l}\text { Weight of Cd. } \\
\text { Gram. }\end{array}$ & $\begin{array}{l}\text { Atomic mass of } \\
\text { cadmium. }\end{array}$ \\
\hline I & 0.43140 & 0.26422 & I 12.054 \\
\hline 2 & $0.4916_{5}$ & 0.30112 & I I $2.05^{2}$ \\
\hline 3 & 0.71752 & 0.43942 & I 12.028 \\
\hline 4 & 0.72188 & 0.44208 & I I $2.02 \mathrm{I}$ \\
\hline 5 & 0.77264 & 0.47319 & I 12.036 \\
\hline 6 & $0.8 \mathrm{I} 224$ & 0.49742 & 112.023 \\
\hline 7 & 0.90022 & 0.55 I 35 & I I 2.04 I \\
\hline 8 & 1.02072 & 0.62505 & I I 2.002 \\
\hline 9 & I. 26322 & 0.77365 & I I 2.04 I \\
\hline Io & I. 52344 & 0.93314 & II 2.078 \\
\hline \multirow{2}{*}{\multicolumn{4}{|c|}{$\begin{aligned} \text { Mean } & =112.038 \\
\text { Maximum } & =112.078\end{aligned}$}} \\
\hline \multirow{2}{*}{\multicolumn{4}{|c|}{ Minimum $=112.07^{\circ}$}} \\
\hline & & & \\
\hline \multicolumn{4}{|c|}{ Difference $=-0.076$} \\
\hline & Probable & $= \pm 0.005$ & \\
\hline
\end{tabular}

and computed for the formula $\mathrm{CdCl}_{2}$, assuming 35.45 to be the atomic mass of chlorine, are as follows:

From the total quantity of material used and metal obtained, we have II 2.040 for the atomic mass of cadmium.

\section{SECOND SERIES.}

PREPARATION OF CADMIUM BROMIDE.

The bromine used in this series was purified as outlined in the experiments on mercuric bromide. The cadmium bromide was prepared by allowing bromine water to act on metallic cadmium for several days at the ordinary temperature. When the action 
was complete, the solution was filtered and evaporated to crystallization. The crystals of cadmium bromide were remored from the liquid and thoroughly dried. The material was then placed in a hard glass combustion tube and carefully sublimed in a current of dry carbon dioxide. The first and last portions of the sublimate were rejected. The middle portion was removed from the tube, placed in a weighing bottle and kept in a desiccator. The product obtained in this way consisted of a crystalline, pearly leaflet which dissolved immediately in water without leaving a residue.

\section{MIODE OF PROCEDURE.}

The method of operation was the same as for cadmium chloride. A weighed portion of the material was dissolved in a little water in a platinum dish. A slight excess of potassium cyanide was then added and after diluting to $200 \mathrm{cc}$. the solution was electrolyzed and the resulting metal weighed. The strength of current and time of action were the same as for cadmium chloride.

Ten observations on cadmium bromide reduced to a vacuum standard on a basis of :

$$
\begin{aligned}
4.8 & =\text { density of cadmium bromide, } \\
8.55 & =\text { " " metallic cadmium, } \\
21.4 & =" \text { " platinum dish, } \\
8.5 & =\text { " " weights, }
\end{aligned}
$$

and computed for the formula $\mathrm{CdBr}_{2}$, assuming 79.95 to be the atomic mass of bromine, are as follows:

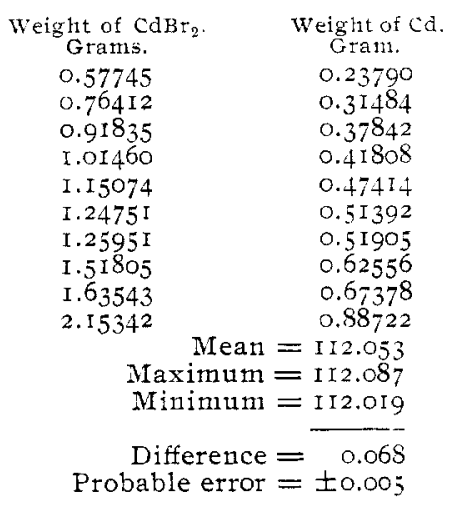

Atomic mass of cadmiun II 2.03 I II 2.052 I I 2.067 I 2.068 II 2.053 II 2.019 II 2.087 I 12.076 I I 2.034 I I $2.04 \mathrm{I}$ 
ATOMIC MASSES OF SILVER, MERCURY AND CADMIUM. IO25

From the total quantity of material used and the metal obtained, $\mathrm{Cd}=\mathrm{I} \mathrm{I} 2.053$.

\section{THIRD SERIES.}

In these experiments an attempt was made to determine the ratio of the atomic mass of cadmium to that of silver by allowing the same electric current to pass successively through solutions of the two metals and weighing the resulting deposits. The arrangement of apparatus and the details of the method were described under the mercury silver series. The results were not as satisfactory as the corresponding results obtained for mercury. A large number of determinations were made with currents of different strength and solutions of different concentration, but the results were, in most cases, far below those obtained in the first two series. A current which deposited about twelve hundredths of a gram of silver per hour seemed to give the best results. From all the observations, five results were selected which differed only about one-tenth of a unit from those of the first two series. Results selected in this way are entitled to but little weight, and perhaps should not be used in determining the general mean of all the observations.

Computed on the basis of 107.92 for the atomic mass of silver, the only admissible results are as follows:

$\begin{array}{cccc}\text { Weight of Ag. } & \text { Weight of Cd. } & \text { Atomic mass } \\ \text { Gram } & \text { Gram. } & \\ \text { I } & 0.24335 & 0.12624 & \text { III.928 } \\ 2 & 0.21262 & 0.11032 & \text { III.99I } \\ 3 & 0.24515 & 0.12720 & \text { III.952 } \\ 4 & 0.2433 \mathrm{I} & 0.12616 & \text { III.9I6 } \\ 5 & 0.42520 & 0.22058 & \text { III.97I } \\ & \text { Mean } & =\text { III.952 } & \\ \text { Maximum } & =\text { III.99I } & \\ \text { Minimum } & =\text { III.9I6 } & \\ \text { Difference } & = & 0.075 & \end{array}$

This method was discussed under mercury. The probable sources of error pointed out there apply equally well in the case of cadmium. Until the large variations can be accounted for 
IO26 ATOMIC MASSES OF SILVER, MERCURY AND CADHIUM.

and the difficulties overcome, the method must be regarded as unsatisfactory,

\section{SCMIIARY.}

Inasmuch as but one method of analysis has been used throughout this work, it is useless to discuss it here. The advantages and objections pointed out under silver apply also to cadmium.

In summing up the work on cadmium, equal weight must be given to the first two series. The last series must be considered alone and all that need be said of it is, that the results obtained for the atomic mass of cadmium never exceeded iI 2 . In the corresponding series on mercury, the variations were in both directions from 200.

The general mean of the first two series calculated from the separate observations is :

$$
\begin{aligned}
& \text { Atomic nass of Cd. } \\
& \text { First series }=\text { II } 2.038 \\
& \text { Second series }=\text { II } 2.053 \\
& \text { General mean }=\overline{\text { II } 2.14455}
\end{aligned}
$$

From the total quantity of material used and metal obtained we have:

$$
\begin{aligned}
& \text { Atomic mass of Cd. } \\
& \text { First series }=I I 2.040 \\
& \text { Second series }=\frac{I I 2.053}{\text { General mean }=}=\operatorname{Ir2.0465}
\end{aligned}
$$

Combining this with the first general mean we have I 2.046 as the most probable result of all the work, for the atomic mass of cadmium. This result is lower than those obtained by Huntington and Bucher, but agrees very closely with the results obtained by von Hauer, Dumas, Lensen, Jones, and Lorimer and Smith.

I wish here to express my sense of gratitude to Professor Edgar F. Smith, at whose suggestion this work was undertaken and under whose personal supervision it was carried out. 